\title{
Image Segmentation using a Fuzzy Roughness Measure
}

\author{
Sheeja T. K, Sunny Kuriakose A.
}

\begin{abstract}
Measures of uncertainty are highly useful for determining the information content of a system. In this paper, new measures of information on fuzzy approximation spaces are introduced based on divergence measures of fuzzy sets. The proposed fuzzy rough uncertainty measure is used to develop an algorithm for histogram based foreground background segmentation of a grey level image and it is experimented with twelve standard test images. It is observed that the overlapping of the foreground background pixels in the images segmented using the proposed method is lesser than those produced by OTSU and FCM methods. The segmented images are compared using their root mean square error values.
\end{abstract}

Index Terms: Rough set, Fuzzy rough set, Uncertainty measure, Image segmentation.

\section{INTRODUCTION}

Image segmentation refers to partitioning of an image into different non-intersecting homogeneous regions. It is a basic process in image analysis. There are many different methodologies that use gray level histogram, spatial details, mathematical morphology, fuzzy set theoretic approaches, neural networks etc. which are successfully applied in image segmentation [3], [8], [7]. Gray level thresholding is a simple and popular method used for the binary segmentation of a gray level image. Some thresholding techniques are described in [5], [18]. OTSU and FCM methods are two classic methods used for image segmentation which are very popular in the image processing research domain [1], [14]. In OTSU method, the threshold is obtained by maximizing the inter-class intensity variance, whereas in FCM method the threshold is determined by optimizing an objective function defined using a measure of similarity between the pixels and the cluster centres.

The theory of rough sets was introduced by Z. Pawlak in early 1980's [15]. It has become a potential tool to manage the uncertainty in data caused by incomplete information. Rough set theory along with its generalized versions have found successful applications in machine learning, decision analysis, artificial intelligence, expert systems, inductive reasoning etc. Information theory, introduced by C. E.

\footnotetext{
Revised Manuscript Received on October 30, 2019.

* Correspondence Author

Sheeja T. K.*, Department of Mathematics, T.M.J.M.Govt. College, Manimalakunnu, India.

Dr. Sunny Kuriakose A.,, Professor and Dean,, FISAT Angamaly, India.
}

(c) The Authors. Published by Blue Eyes Intelligence Engineering and Sciences Publication (BEIESP). This is an open access article under the CC BY-NC-ND license (http://creativecommons.org/licenses/by-nc-nd/4.0/)
Shannon [19], studies the transmission, processing, extraction, and utilization of information. Uncertainty measures play a vital role in information theory as means for quantifying the capacity of a system to process information.

Many attempts are there in the literature, to define uncertainty measures in the context of rough sets and fuzzy rough sets [10], [20], [23]. Studies on the applications of rough set concepts to image processing are given in [6]. In this paper, new uncertainty measures on fuzzy approximation spaces are defined based on fuzzy divergence measures. Divergence measures provide an indication of the extent of difference between two fuzzy sets [13]. It is shown that these new measures are generalizations of some of the existing uncertainty measures on crisp and fuzzy approximation spaces. Further, a histogram based image thresholding algorithm using the fuzzy roughness measure is presented and experimented with different images. The segmented images of twelve standard test images namely, baboon, barbara, boat, bridge, cameraman, house, lena, mountain, peppers, rice, sailboat and zelda are compared with those of OTSU method and FCM method. It is observed that the overlapping of the foreground background pixels in the images segmented using the proposed method is lesser than those produced by the OTSU and FCM methods. Further, the proposed algorithm produces images having the least root mean square error values in ten out of twelve cases. The remaining part of the paper is organized as follows: some basic concepts related to this paper are recalled in section 2, section 3 presents the new fuzzy rough uncertainty measures, section 4 describes an application of the proposed measure to image segmentation and conclusion is given in section 5 .

\section{BASIC CONCEPTS}

Consider a non-empty finite set of objects denoted by $U$. Let $\Theta$ be a fuzzy equivalence relation on $U$.

Definition 2.1. [9] The fuzzy equivalence classes of $\Theta$ are defined $\forall u \in U$ as, $\mu_{[u]_{\Theta}}(v)=\Theta(u, v), \quad \forall v \in U$.

Definition 2.2. [22] The fuzzy cardinality of a fuzzy subset $X$ of $U$ is given by, $|X|=\sum_{u \in U} X(u)$

Definition 2.3. [4] A weak fuzzy partition on $U$ is a collection $\left\{A_{1}, A_{2}, \ldots, A_{k}\right\}$ of fuzzy sets on $U$ such that $\inf _{u}\left(\max _{i} A_{i}(u)>0\right.$ and $\sup _{u}\left(\min \left(A_{i}(u), A_{j}(u)\right)<1, \forall i, j\right.$. Definition 2.4. [13] A function $\delta: \mathcal{F}(U) \times \mathcal{F}(U) \rightarrow R$ is said to be a divergence measure iff $\forall X, Y, Z \in \mathcal{F}(U)$,

$$
\begin{array}{ll}
\text { i. } & \delta(X, X)=0 \\
\text { ii. } & \delta(X, Y)=\delta(Y, X) \\
\text { iii. } & \max (\delta(X \cup Z, Y \cup Z), \delta(X \cap Z, Y \cap Z)) \leq \\
& \delta(X, Y) \\
\text { Published By: } & \text { Blue Eyes Intelligence Engineering } \\
\text { \& Sciences Publication }
\end{array}
$$


where, $\mathcal{F}(U)$ is the collection of all fuzzy sets on $U$.

Proposition 2.5. [13] For the fuzzy subsets $X, Y, Z, W$ of $\mathrm{U}$ such that $X \subseteq Y \subseteq Z \subseteq W, \delta(Y, Z) \leq \delta(X, W)$

Definition 2.6. [15] Let $\theta$ be a crisp equivalence relation on $\mathrm{U}$. Then, the lower approximation and the upper approximation of $X \subseteq U$ with respect to $\theta$ are respectively defined as

$$
\begin{gathered}
\underline{\theta}(X)=\left\{u \in U:[u\}_{\theta} \subseteq X\right\} \text { and } \bar{\theta}(X)=\{u \in U: \\
\left.[u\}_{\theta} \cap X \neq \Phi\right\} .
\end{gathered}
$$

Definition 2.7. [16] The $\theta$ - roughness of $X \subseteq U$ is given by

$$
\rho_{\theta}(X)=1-\frac{\theta}{\overline{\bar{\theta}}(X)}
$$

Definition 2.8. [20] Consider $U / \theta=\left\{\theta_{1}, \theta_{2}, \ldots, \theta_{k}\right\}$. An information measure of $(U, \theta)$ is given by

$$
G(\theta)=-\sum_{i=1}^{k} \frac{\left|\theta_{i}\right|}{|U|} \log _{2} \frac{\left|\theta_{i}\right|}{|U|}
$$

Definition 2.9. [4] The fuzzy rough lower and upper approximations of a fuzzy set $X$ on $U$ are given by

$$
\begin{gathered}
\mu_{\underline{\Theta}(X)}(u)=\inf _{v \in U} \max \left(1-\Theta(u, v), \mu_{X}(v)\right) \\
\mu_{\bar{\Theta}(X)}(u)=\sup _{v \in U} \min \left(\Theta(u, v), \mu_{X}(v)\right)
\end{gathered}
$$

respectively.

There are several intensive studies on the theory and applications of fuzzy rough sets [12], [17], [21]. A survey of the various approaches to fuzzy rough sets was conducted by L. Deer et al [2].

Definition 2.10. [11] Let $U=\left\{u_{1}, u_{2}, \ldots, u_{n}\right\}$. The entropy of the fuzzy approximation space $(U, \Theta)$ is given by,

\section{INFORMATION MEASURES ON FUZZY APPROXIMATION SPACES}

Let $U=\left\{u_{1}, u_{2}, \ldots, u_{n}\right\}$. Let $[u i]_{\Theta}$ represents a fuzzy equivalence class of $(U, \Theta)$. That is, $[u i]_{\Theta}: U \rightarrow[0,1]$, $\mu_{\left[u_{i}\right]_{\Theta}}(v)=\Theta\left(u_{i}, u_{j}\right), \forall u_{j} \in U$. Also, let $\widehat{U}$ be the fuzzy set $\widehat{U}: U \rightarrow[0,1], \widehat{U}\left(u_{i}\right)=1, \forall u_{i} \in U$ and $\widehat{\varnothing}$ be the fuzzy set $\widehat{\emptyset}: U \rightarrow[0,1], \widehat{\varnothing}\left(u_{i}\right)=0, \forall u_{i} \in U$. Let $\delta(X, Y)$ be a divergence measure on $\mathcal{F}(U) \times \mathcal{F}(U)$. For notational convenience, we assume that $\log _{2}(0)=0$.

Definition 3.1. For the fuzzy approximation space $(U, \Theta)$, the $\delta$-information entropy is defined as

$$
E_{\delta}(\Theta)=-\frac{1}{|U|} \sum_{i=1}^{n} \log _{2}\left(1-\frac{\delta\left(\widehat{U},[u i]_{\Theta}\right)}{\delta(\widehat{U}, \widehat{\varnothing})}\right)
$$

Proposition 3.2 On a fuzzy approximation space $(U, \Theta)$, if $\Theta\left(u_{i}, u_{j}\right)=1, \forall u_{i}, u_{j} \in U$, then $E_{\delta}(\Theta)=0$.

Proof:

$$
\begin{gathered}
\Theta\left(u_{i}, u_{j}\right)=1, \forall u_{i}, u_{j} \in U \Rightarrow[u i]_{\Theta}=\widehat{U}, \forall u_{i} \in U \\
\Rightarrow \delta\left(\widehat{U},[u i]_{\Theta}\right)=\delta(\widehat{U}, \widehat{U})=0, \forall u_{i} \in U \\
\Rightarrow \log _{2}\left(1-\frac{\delta\left(\widehat{U},[u i]_{\Theta}\right)}{\delta(\widehat{U}, \widehat{\varnothing})}\right)=\log _{2}\left(1-\frac{0}{\delta(\widehat{U}, \widehat{\varnothing})}\right) \\
\quad=\log _{2} 1=0, \forall u_{i} \in U
\end{gathered}
$$

Hence, from equation (1), $E_{\delta}(\Theta)=0$.

Now, we will show that the information measure on a crisp approximation space defined by Wiermann [20] is a special case of the proposed $\delta$-information entropy measure.

Theorem 3.3 Consider a crisp approximation space $(U, \theta)$. Then, $E_{\delta}(\theta)=G(\theta)$ if $\delta(X, Y)=\sum_{j=1}^{n}\left|\mathrm{X}\left(\mathrm{u}_{\mathrm{i}}\right)-\mathrm{Y}\left(\mathrm{u}_{\mathrm{i}}\right)\right|$.

Proof:

Let $U / \theta=\left\{\theta_{1}, \theta_{2}, \ldots, \theta_{k}\right\}$.Then, each $u \in U$ is an element of exactly one equivalence class. We have,

$$
F(\Theta)=-\sum_{i=1}^{n} \frac{1}{|U|} \log _{2} \frac{\left|[u i]_{\Theta}\right|}{|U|}
$$

$$
\theta\left(u_{i}, u_{j}\right)= \begin{cases}1, & \text { if } u_{j} \in[u i]_{\theta} \\ 0, & \text { otherwise }\end{cases}
$$

Hence, $\delta\left(\widehat{U},[u i]_{\theta}=\sum_{j=1}^{n}\left|\widehat{U}\left(\mathrm{u}_{\mathrm{j}}\right)-[u i]_{\theta}\left(\mathrm{u}_{\mathrm{j}}\right)\right|\right.$

$$
\begin{aligned}
& =\sum_{j=1}^{n}\left|1-\theta\left(\mathrm{u}_{1}, \mathrm{u}_{\mathrm{j}}\right)\right|=\sum_{u_{j} \notin[u i]_{\theta}} 1 \\
& =\left|\left([u i]_{\theta}\right)^{C}\right|=|U|-\left|[u i]_{\theta}\right|
\end{aligned}
$$

Also, $\delta(\widehat{U}, \widehat{\emptyset})=\sum_{j=1}^{n}\left|\widehat{U}\left(\mathrm{u}_{\mathrm{j}}\right)-\widehat{\emptyset}\left(\mathrm{u}_{\mathrm{j}}\right)\right|$

$$
=\sum_{j=1}^{n}|1-0|=|U|
$$

Therefore, $E_{\delta}(\theta)=-\frac{1}{|U|} \sum_{i=1}^{n} \log _{2}\left(1-\frac{\delta\left(\widehat{U},[u i]_{\theta}\right)}{\delta(\widehat{U}, \widehat{\varnothing})}\right)$

$$
\begin{aligned}
& =-\frac{1}{|U|} \sum_{i=1}^{n} \log _{2}\left(1-\frac{\left.|U|-\left|[u i]_{\theta}\right|\right)}{|U|}\right) \\
& =-\sum_{i=1}^{n} \frac{1}{|U|} \log _{2}\left(\frac{\left.\left|[u i]_{\theta}\right|\right)}{|U|}\right)
\end{aligned}
$$

Let $[u i]_{\Theta}=\theta_{j}$. Then, in the above sum, each term $\frac{1}{|U|} \log _{2}\left(\frac{\left.\left|[u i]_{\Theta}\right|\right)}{|U|}\right)$ is repeated $\theta_{j}$ times. Hence, it follows that, $E_{\delta}(\theta)=-\sum_{i=1}^{k} \frac{\left|\theta_{j}\right|}{|U|} \log _{2}\left(\frac{\left|\theta_{j}\right|}{|U|}\right)=G(\theta)$.

The fact that the fuzzy rough entropy proposed by J. S. Mi et al. [11] is a special case of the proposed $\delta$-information entropy measure is asserted in the next theorem.

Theorem 3.4 On a fuzzy approximation space $(U, \Theta)$, $E_{\delta}(\Theta)=F(\Theta)$, for $\delta(X, Y)=\sum_{j=1}^{n}\left|\mathrm{X}\left(\mathrm{u}_{\mathrm{i}}\right)-\mathrm{Y}\left(\mathrm{u}_{\mathrm{i}}\right)\right|$

Proof:

$$
\begin{aligned}
& E_{\delta}(\theta)=-\frac{1}{|U|} \sum_{i=1}^{n} \log _{2}\left(1-\frac{\delta\left(\widehat{U},[u i]_{\Theta}\right)}{\delta(\widehat{U}, \widehat{\varnothing})}\right) \\
& =-\frac{1}{|U|} \sum_{i=1}^{n} \log _{2}\left(1-\frac{\sum_{j=1}^{n}\left|\widehat{U}\left(\mathrm{u}_{\mathrm{j}}\right)-[u i]_{\Theta}\left(\mathrm{u}_{\mathrm{j}}\right)\right|}{\sum_{j=1}^{n}\left|\widehat{U}\left(\mathrm{u}_{\mathrm{j}}\right)-\widehat{\emptyset}\left(\mathrm{u}_{\mathrm{j}}\right)\right|}\right) \\
& =-\frac{1}{|U|} \sum_{i=1}^{n} \log _{2}\left(1-\frac{\sum_{j=1}^{n}\left|1-\Theta\left(u_{i}, u_{j}\right)\right|}{\sum_{j=1}^{n}|1-0|}\right) \\
& =-\frac{1}{|U|} \sum_{i=1}^{n} \log _{2}\left(1-\frac{|U|-\sum_{j=1}^{n} \Theta\left(u_{i}, u_{j}\right)}{|U|}\right) \\
& =-\frac{1}{|U|} \sum_{i=1}^{n} \log _{2}\left(\frac{\sum_{j=1}^{n} \Theta\left(u_{i}, u_{j}\right)}{|U|}\right) \\
& =-\frac{1}{|U|} \sum_{i=1}^{n} \log _{2}\left(\frac{\left.\left|[u i]_{\theta}\right|\right)}{|U|}\right)=F(\Theta)
\end{aligned}
$$

The monotonic property of the $\delta$-information entropy measure is presented in the theorem given below.

Theorem 3.5 If $\Theta_{1}$ and $\Theta_{2}$ are fuzzy equivalence relations on $U$ and $\Theta_{1} \subseteq \Theta_{2}$, then $E_{\delta}\left(\theta_{1}\right) \geq E_{\delta}\left(\theta_{2}\right)$.

Proof:

$$
\begin{aligned}
& \Theta_{1} \subseteq \Theta_{2} \Rightarrow \Theta_{1}\left(u_{i}, u_{j}\right) \leq \Theta_{2}\left(u_{i}, u_{j}\right), \forall u_{i}, u_{j} \in U \\
& \Rightarrow[u i]_{\Theta_{1}}\left(u_{j}\right) \leq[u i]_{\Theta_{2}}\left(u_{j}\right), \forall u_{i}, u_{j} \in U \\
& \Rightarrow[u i]_{\Theta_{1}} \subseteq[u i]_{\Theta_{2}} \subseteq \widehat{U}, \forall u_{i} \in U \\
& \Rightarrow \delta\left(\widehat{U},[u i]_{\Theta_{1}}\right) \geq \delta\left(\widehat{U},[u i]_{\Theta_{2}}\right), \forall u_{i} \in U \\
& \Rightarrow \frac{\delta\left(\widehat{U},[u i]_{\Theta_{1}}\right)}{\delta(\widehat{U}, \widehat{\varnothing})} \geq \frac{\delta\left(\widehat{U},[u i]_{\Theta_{2}}\right)}{\delta(\widehat{U}, \widehat{\varnothing})} \\
& \Rightarrow \log _{2}\left(1-\frac{\delta\left(\widehat{U},[u i]_{\Theta_{1}}\right)}{\delta(\widehat{U}, \widehat{\varnothing})}\right) \leq \log _{2}\left(1-\frac{\delta\left(\widehat{U},[u i]_{\Theta_{2}}\right)}{\delta(\widehat{U}, \widehat{\varnothing})}\right) \\
& \Rightarrow E_{\delta}\left(\Theta_{1}\right) \geq E_{\delta}\left(\Theta_{2}\right)
\end{aligned}
$$

Definition 3.6 The joint $\delta$-information entropy of the fuzzy equivalence relations $\Theta_{1}$ and $\Theta_{2}$ on $U$ is defined as

$$
E_{\delta}\left(\Theta_{1}: \Theta_{2}\right)=-\frac{1}{|U|} \sum_{i=1}^{n} \log _{2}\left(1-\frac{\delta\left(\widehat{U},[u i]_{\Theta_{1}} \cap[u i]_{\Theta_{2}}\right)}{\delta(\widehat{U}, \widehat{\emptyset})}\right)
$$

Definition 3.7 The conditional $\delta$-information entropy of the fuzzy equivalence relation $\Theta_{1}$ given $\Theta_{2}$ is defined to be

$$
E_{\delta}\left(\Theta_{1} \mid \Theta_{2}\right)=-\frac{1}{|U|} \sum_{i=1}^{n} \log _{2}\left(\frac{\delta(\widehat{U}, \widehat{\varnothing})-\delta\left(\widehat{U},[u i]_{\Theta_{1}} \cap[u i]_{\Theta_{2}}\right)}{\delta(\widehat{U}, \widehat{\varnothing})-\delta\left(\widehat{U},[u i]_{\Theta_{2}}\right)}\right)(3)
$$

Theorem 3.8 For two fuzzy equivalence relations $\Theta_{1}$ and $\Theta_{2}$ on $\mathrm{U}, E_{\delta}\left(\Theta_{1} \mid \Theta_{2}\right)=$ $E_{\delta}\left(\Theta_{1}: \Theta_{2}\right)-E_{\delta}\left(\Theta_{2}\right)$

Proof: 
We have, $E_{\delta}\left(\Theta_{1}: \Theta_{2}\right)-E_{\delta}\left(\Theta_{2}\right)=$

$$
\begin{aligned}
& -\frac{1}{|U|} \sum_{i=1}^{n} \log _{2}\left(1-\frac{\delta\left(\widehat{U},[u i]_{\Theta_{1}} \cap[u i]_{\Theta_{2}}\right)}{\delta(\widehat{U}, \widehat{\varnothing})}\right)- \\
& -\frac{1}{|U|} \sum_{i=1}^{n} \log _{2}\left(1-\frac{\delta\left(\widehat{U},[u i]_{\Theta_{2}}\right)}{\delta(\widehat{U}, \widehat{\emptyset})}\right)= \\
& -\frac{1}{|U|} \sum_{i=1}^{n} \log _{2} \frac{1-\frac{\delta\left(\widehat{U},[u i]_{\Theta_{1}} \cap[u i]_{\Theta_{2}}\right)}{\delta(\widehat{U}, \widehat{\phi})}}{1-\frac{\delta\left(\widehat{U},[u i]_{\Theta_{2}}\right)}{\delta(\widehat{U}, \widehat{\varnothing})}}
\end{aligned}
$$

$=-\frac{1}{|U|} \sum_{i=1}^{n} \log _{2}\left(\frac{\delta(\widehat{U}, \widehat{\varnothing})-\delta\left(\widehat{U},[u i]_{\Theta_{1}} \cap[u i]_{\Theta_{2}}\right)}{\delta(\widehat{U}, \widehat{\varnothing})-\delta\left(\widehat{U},[u i]_{\Theta_{2}}\right)}\right)=E_{\delta}\left(\Theta_{1} \mid \Theta_{2}\right)$

Definition 3.9 Let $\Theta_{1}$ and $\Theta_{2}$ be fuzzy equivalence relations on . Then the $\delta$-mutual information of $\Theta_{1}$ and $\Theta_{2}$ is defined as $I_{\delta}\left(\Theta_{1}: \Theta_{2}\right)=$

$-\frac{1}{|U|} \sum_{i=1}^{n} \log _{2}\left(\frac{\left(\delta(\widehat{U}, \widehat{\emptyset})-\delta\left(\widehat{U},[u i]_{\Theta_{1}}\right)\right)\left(\delta(\widehat{U}, \widehat{\emptyset})-\delta\left(\widehat{U},[u i]_{\Theta_{2}}\right)\right)}{\delta(\widehat{U}, \widehat{\varnothing})\left(\delta(\widehat{U}, \widehat{\varnothing})-\delta\left(\widehat{U},[u i]_{\Theta_{1}} \cap[u i]_{\Theta_{2}}\right)\right)}\right)$

Theorem 3.10 If $\Theta_{1}$ and $\Theta_{2}$ are fuzzy equivalence relations on , then $I_{\delta}\left(\Theta_{1}: \Theta_{2}\right)=E_{\delta}\left(\Theta_{1}\right)-E_{\delta}\left(\Theta_{1} \mid \Theta_{2}\right)$

Proof:

We have, $E_{\delta}\left(\Theta_{1}\right)-E_{\delta}\left(\Theta_{1} \mid \Theta_{2}\right)=$

$-\frac{1}{|U|} \sum_{i=1}^{n} \log _{2}\left(1-\frac{\delta\left(\widehat{U},[u i]_{\Theta_{1}}\right)}{\delta(\widehat{U}, \widehat{\varnothing})}\right)-$

$$
-\frac{1}{|U|} \sum_{i=1}^{n} \log _{2}\left(\frac{\delta(\widehat{U}, \widehat{\varnothing})-\delta\left(\widehat{U},[u i]_{\Theta_{1}} \cap[u i]_{\Theta_{2}}\right)}{\delta(\widehat{U}, \widehat{\varnothing})-\delta\left(\widehat{U},[u i]_{\Theta_{2}}\right)}\right)
$$

$=-\frac{1}{|U|} \sum_{i=1}^{n} \log _{2}\left(\frac{\frac{\delta(\widehat{U}, \widehat{\phi})-\delta\left(\widehat{U},[u i]_{\Theta_{1}}\right)}{\delta(\widehat{U}, \widehat{\phi})}}{\frac{\delta(\widehat{U}, \widehat{\phi})-\delta\left(\widehat{U},[u i]_{\Theta_{1}} \cap[u i]_{\Theta_{2}}\right)}{\delta(\widehat{U}, \widehat{\phi})-\delta\left(\widehat{U},[u i]_{\Theta_{2}}\right)}}\right)$

$=-\frac{1}{|U|} \sum_{i=1}^{n} \log _{2}\left(\frac{\left(\delta(\widehat{U}, \widehat{\varnothing})-\delta\left(\widehat{U},[u i]_{\Theta_{1}}\right)\right)\left(\delta(\widehat{U}, \widehat{\varnothing})-\delta\left(\widehat{U},[u i]_{\Theta_{2}}\right)\right)}{\delta(\widehat{U}, \widehat{\varnothing})\left(\delta(\widehat{U}, \widehat{\varnothing})-\delta\left(\widehat{U},[u i]_{\Theta_{1}} \cap[u i]_{\Theta_{2}}\right)\right)}\right)$

$=I_{\delta}\left(\Theta_{1}: \Theta_{2}\right)$

In the following, we propose a fuzzy roughness measure of a fuzzy set using the divergence measure $\delta$ and prove that it generalizes Pawlak's roughness measure [16].

Definition 3.11 Let $(U, \Theta)$ be a fuzzy approximation space. Then the $\delta$-fuzzy roughness measure of a fuzzy set $X \neq \varnothing$ on $U$ is defined as

$$
E_{\delta}^{\Theta}(X)=\frac{\delta(\bar{\Theta}(\mathrm{X}), \underline{\Theta}(\mathrm{X}))}{\delta(\bar{\Theta}(\mathrm{X}), \widehat{\emptyset})}
$$

Theorem 3.12 The $\delta$-fuzzy roughness measure of a crisp set $X$ on a crisp approximation space $(U, \theta)$ is the same as the roughness of $X$ with respect to $\theta$, when $\delta(X, Y)=$ $\sum_{j=1}^{n}\left|\mathrm{X}\left(\mathrm{u}_{\mathrm{j}}\right)-\mathrm{Y}\left(\mathrm{u}_{\mathrm{j}}\right)\right|$

Proof:

We have, $\theta\left(u_{i}, u_{j}\right)=\left\{\begin{array}{lr}1, & \text { if } u_{j} \in[u i]_{\theta} \\ 0, & \text { otherwise }\end{array}\right.$

Hence, $\delta(\bar{\Theta}(\mathrm{X}), \underline{\Theta}(\mathrm{X}))=\sum_{j=1}^{n}\left|\bar{\Theta}(\mathrm{X})\left(\mathrm{u}_{\mathrm{j}}\right)-\underline{\Theta}(\mathrm{X})\left(\mathrm{u}_{\mathrm{j}}\right)\right|$

$=\sum_{j=1}^{n} \bar{\Theta}(\mathrm{X})\left(\mathrm{u}_{\mathrm{j}}\right)-\sum_{j=1}^{n} \underline{\Theta}(\mathrm{X})\left(\mathrm{u}_{\mathrm{j}}\right)$ since, $\underline{\Theta}(\mathrm{X}) \subseteq \bar{\Theta}(\mathrm{X})$

$=|\bar{\Theta}(\mathrm{X})|-|\underline{\Theta}(\mathrm{X})|$

Also, $\delta(\bar{\Theta}(\mathrm{X}), \widehat{\varnothing})=\sum_{j=1}^{n}\left|\bar{\Theta}(\mathrm{X})\left(\mathrm{u}_{\mathrm{j}}\right)-\widehat{\varnothing}\left(\mathrm{u}_{\mathrm{j}}\right)\right|$

$=\sum_{j=1}^{n} \bar{\Theta}(\mathrm{X})\left(\mathrm{u}_{\mathrm{j}}\right)=|\bar{\Theta}(\mathrm{X})|$

Therefore, $E_{\delta}^{\Theta}(X)=\frac{\delta(\bar{\Theta}(\mathrm{X}), \underline{\Theta}(\mathrm{X}))}{\delta(\bar{\Theta}(\mathrm{X}), \widehat{\varnothing})}=\frac{|\bar{\Theta}(\mathrm{X})|-|\underline{\Theta}(\mathrm{X})|}{|\bar{\Theta}(\mathrm{X})|}$

$=1-\frac{|\underline{\Theta}(\mathrm{X})|}{|\bar{\Theta}(\mathrm{X})|}=\rho_{\theta}(X)$

Definition 3.12 Let $(U, \Theta)$ be a fuzzy approximation space. then, a fuzzy set $X$ on $U$ is said to be

i. $\Theta$-definable if $\underline{\Theta}(\mathrm{X})=\bar{\Theta}(\mathrm{X})$ ii. roughly $\Theta$-definable if $\underline{\Theta}(\mathrm{X}) \neq \widehat{\emptyset}$ and $\bar{\Theta}(\mathrm{X}) \neq \widehat{U}$

iii. internally $\Theta$-indefinable if $\underline{\Theta}(\mathrm{X})=\widehat{\varnothing}$ and $\bar{\Theta}(\mathrm{X}) \neq \widehat{U}$

iv. externally $\Theta$-indefinable if $\underline{\Theta}(\mathrm{X}) \neq \widehat{\varnothing}$ and $\bar{\Theta}(\mathrm{X})=\widehat{U}$

v. totally $\Theta$-indefinable if $\underline{\Theta}(\mathrm{X})=\widehat{\emptyset}$ and $\bar{\Theta}(\mathrm{X})=\widehat{U}$

Theorem 3.13 Let $(U, \Theta)$ be a fuzzy approximation space. Then, $\forall X \in \mathcal{F}(U)$,
i. $\quad 0 \leq E_{\delta}^{\Theta}(X) \leq 1$
ii. $E_{\delta}^{\Theta}(X)=0$, if $X$ is $\Theta$-definable
iii. $E_{\delta}^{\Theta}(X)=1$, if $X$ is totally $\Theta$-indefinable

Proof:

i. We have, $\widehat{\varnothing} \subseteq \underline{\Theta}(X) \subseteq \bar{\Theta}(X) \subseteq \widehat{U}$. Hence,

$\delta(\bar{\Theta}(\mathrm{X}), \underline{\Theta}(\mathrm{X})) \leq \delta(\bar{\Theta}(\mathrm{X}), \widehat{\varnothing})$, by proposition 2.5 .

Therefore, $0 \leq \frac{\delta(\bar{\Theta}(\mathrm{X}), \underline{\Theta}(\mathrm{X}))}{\delta(\bar{\Theta}(\mathrm{X}), \widehat{\varnothing})} \leq 1$.

That is, $0 \leq E_{\delta}^{\Theta}(X) \leq 1$

ii. If $X$ is a $\Theta$-definable fuzzy set, then $\underline{\Theta}(X)=\bar{\Theta}(X)$.

Hence, $\delta(\bar{\Theta}(\mathrm{X}), \underline{\Theta}(\mathrm{X}))=0$.

Therefore, $E_{\delta}^{\Theta}(X)=\frac{\delta(\bar{\Theta}(\mathrm{X}), \underline{\Theta}(\mathrm{X}))}{\delta(\bar{\Theta}(\mathrm{X}), \widehat{\phi})}=0$.

iii. If $X$ is a totally $\Theta$-indefinable fuzzy set, then

$\underline{\Theta}(\mathrm{X})=\widehat{\emptyset}$ and $\bar{\Theta}(\mathrm{X})=\widehat{U}$.

Hence, $E_{\delta}^{\Theta}(X)=\frac{\delta(\bar{\Theta}(\mathrm{X}), \underline{\Theta}(\mathrm{X}))}{\delta(\bar{\Theta}(\mathrm{X}), \widehat{\varnothing})}=\frac{\delta(\widehat{U}, \widehat{\varnothing})}{\delta(\widehat{U}, \widehat{\varnothing})}=1$.

Remark:Definition 3.11 is also applicable to generalized fuzzy rough sets as it depends only on the fuzzy rough approximations.

\section{IMAGE SEGMENTATION USING THE FUZZY ROUGHNESS MEASURE}

Image segmentation is a major area of research in digital image processing. Object background segmentation falls under the category of region based image segmentation. In this section, the proposed fuzzy rough uncertainty measure is applied in object background segmentation using grey level thresholding. Here, global thresholding is done using the grey level histogram of the entire image.

Consider a grey level image of order $m \times n$. Let $G$ denote the collection of all pixel values in it. Then, $G=$ $\{0,1, \ldots, 255\}$. First, we divide the set of grey level values $G$ into $N$ parts and obtain the most frequent grey level value (say $v_{k}$ ) in each part by using the histogram of the given image. Corresponding to each $v_{k}$, for $k=1,2, \ldots, N$, we define a fuzzy set $A_{k}$ on $G$, as

$$
A_{k}(i)=\frac{\mid g(i)-g\left(v_{k} \mid\right.}{\max \left(g(i), g\left(v_{k}\right)\right)}
$$

Then, $P=\left\{A_{k}: k=1,2, \ldots, N\right\}$ forms a weak fuzzy partition on $G$. We define an $Z$-shaped fuzzy membership function on $G$ as $F_{G}: G \rightarrow[0,1]$,

$$
F_{G}(i)=\left\{\begin{array}{c}
1, \quad \text { if } \quad i<a \\
\frac{b-i}{b-a}, \quad \text { if } a \leq i \leq b \\
0, \quad \text { otherwise }
\end{array}\right.
$$

For each gray level value $s$, we define a fuzzy set corresponding to the object in the image as

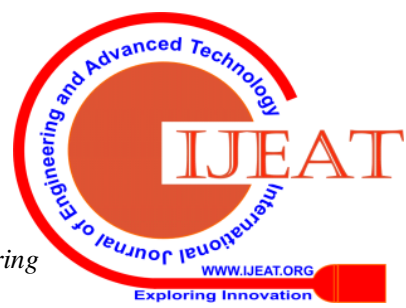




$$
O_{s}(i)=\left\{\begin{array}{l}
F_{G}(i) \text { if } i<s \\
F_{G}(s) \text { if } i \geq s
\end{array}\right.
$$

Let $\underline{\mathrm{P}}\left(O_{s}\right)$ and $\overline{\mathrm{P}}\left(O_{s}\right)$ denote the fuzzy rough approximations of $O_{s}$ corresponding to the weak fuzzy partition P. That is;

$\underline{\mathrm{P}}\left(O_{s}\right)=\sup _{k}\left\{\min _{i}\left[A_{k}(i), \inf _{i}\left(\max \left(1-A_{k}(i), O_{s}(i)\right)\right]\right\}(9)\right.$

$\overline{\mathrm{P}}\left(O_{s}\right)=\sup _{k}\left\{\min _{i}\left[A_{k}(i), \sup _{i}\left(\max \left(A_{k}(i), O_{s}(i)\right)\right]\right\}\right.$

The fuzzy roughness measure corresponding to $s$ is given by

$G(s)=\frac{\delta\left(\overline{\mathrm{P}}\left(O_{s}\right), \underline{\mathrm{P}}\left(O_{s}\right)\right)}{\delta\left(\overline{\mathrm{P}}\left(O_{s}\right), \widehat{\phi}\right)}$

Here, we use the divergence measure given by $\delta(X, Y)=$ $\sum_{u \in U} h(X(u), Y(u))$, where $h$ represents the Kullback Leibler symmetrical function [13]. The value of $s$ that makes the fuzzy roughness measure the minimum is selected as the threshold $c$. If the minimum is obtained for more than one value of $s$, then the largest of such values of $s$ is selected as $c$. Then the image is segmented into a binary image.

\section{Algorithm}

Step1: Input the grey level image $G$

Step2: Find the weak fuzzy partition $\mathrm{P}$

Step3: For s=1:256, find the fuzzy set $O_{s}$ (eqn 8)

Step4: Find the fuzzy rough approximations of $O_{s}$ (eqns 9 and 10)

Step5: Find the fuzzy roughness measure $G(s)$ of $O_{s}$ (eqn 11) Step6: Find the value c of $s$ for which $G$ is the minimum Step7: Segment the image into a binary one using $c$

\section{Experimental Results}

The histogram based image thresholding algorithm for object background segmentation using the fuzzy roughness measure is implemented using twelve standard test images namely, baboon, barbara, boat, bridge, cameraman, house, lena, mountain, peppers, rice, sailboat and zelda. The original images and the segmented ones using the proposed method, OTSU method and FCM method are presented in figures $1-12$.

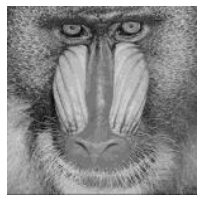

(a) Input

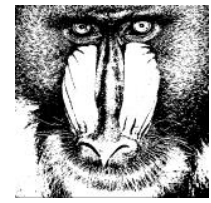

(b) proposed

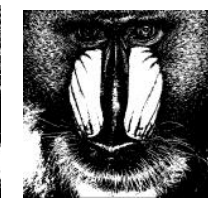

(c) FCM

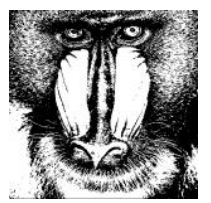

(d) Otsu
Figure 1: Baboon

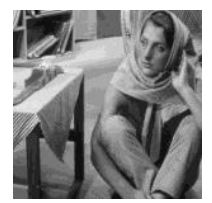

(a) Input

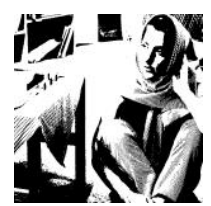

(b) proposed

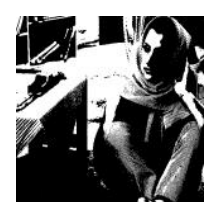

(c) FCM

Figure 2: Barbara

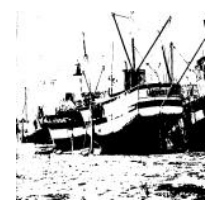

(b) proposed

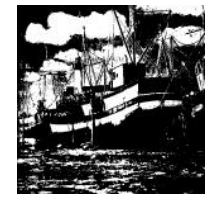

(c) FCM

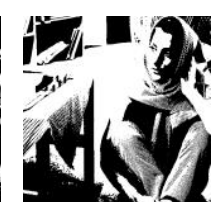

(d) Otsu

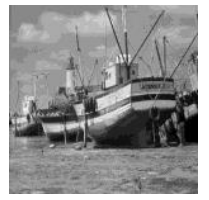

(a) Input
Figure 3: Boat (d) Otsu

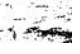

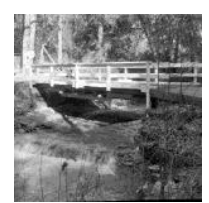

(a) Input

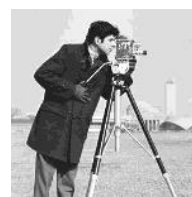

(a) Input

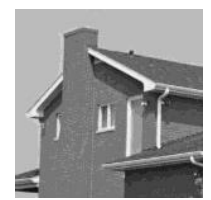

(a) Input

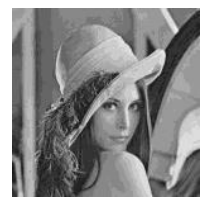

(a) Input

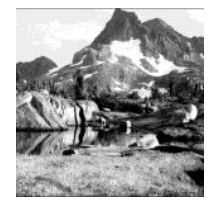

(a) Input

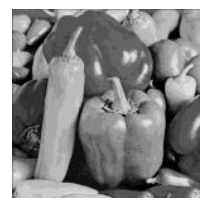

(a) Input

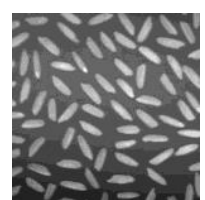

(a) Input

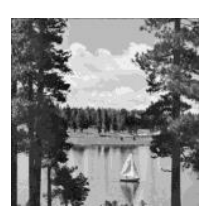

(a) Input

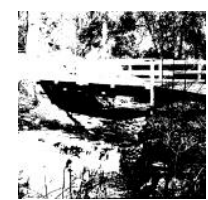

(b) proposed

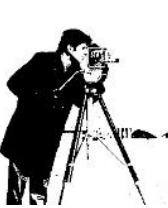

(b) proposed

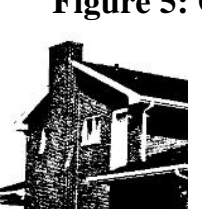

(b) proposed

Figure 6: House

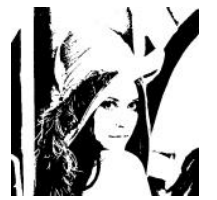

(b) proposed

Figure 7: Lena

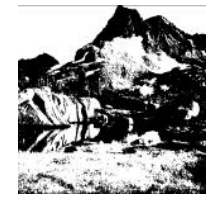

(b) proposed

Figure 8: Mountain

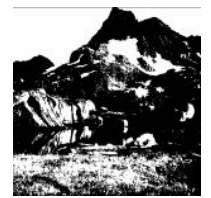

(c) FCM
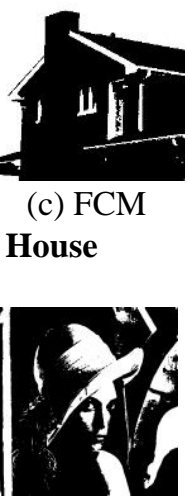

(c) FCM

(d) Otsu

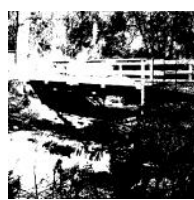

(d) Otsu c) FCM

c) FCM

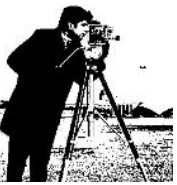

(d) Otsu

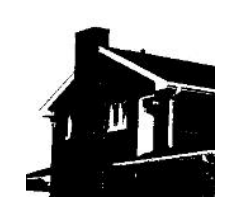

(d) Otsu
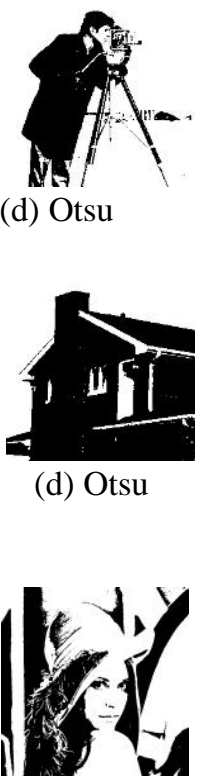

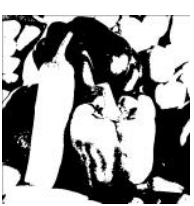

(b) proposed

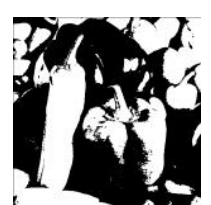

(c) FCM

Figure 9: Peppers

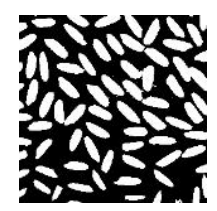

(b) proposed

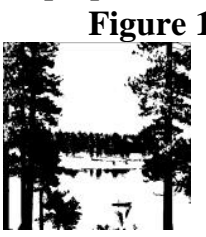

(b) proposed

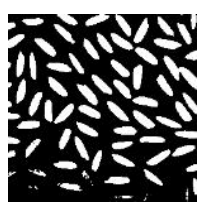

(c) FCM

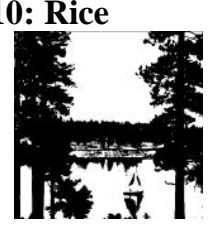

(c) FCM

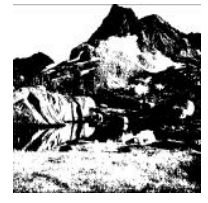

(d) Otsu

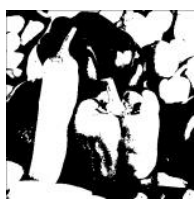

(d) Otsu

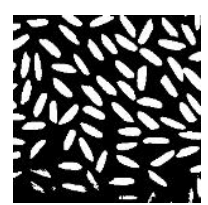

(d) Otsu

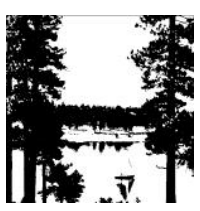

(d) Otsu

Figure 11: Sailboat

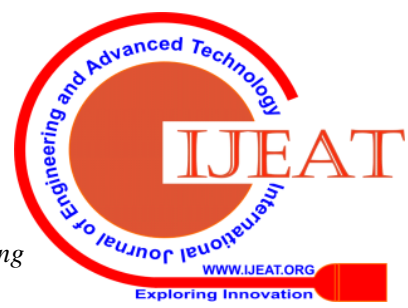




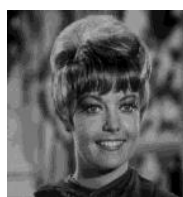

(a) Input

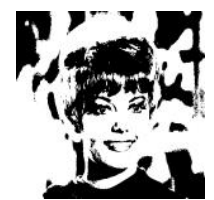

(b) proposed

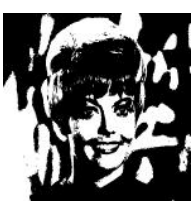

(c) FCM

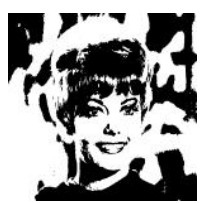

(d) Otsu

\section{Figure 12: Zelda}

It is observed that the proposed method produces clear binary images in all the twelve instances. It is clear from the figures that the overlapping of the foreground background pixels in the images segmented using the proposed method is lesser than those of the other two methods in most of the cases. Moreover, the RMSE values of the segmented images using the proposed algorithm are found to be the least in comparison with those segmented by OTSU and FCM methods. The values are presented in table 1 .

Table 1: RMSE Values

\begin{tabular}{|l|l|l|l|l|}
\hline Sl.No. & Image & Proposed & FCM & OTSU \\
\hline 1 & Baboon & $\mathbf{1 0 . 4 9 1 3}$ & 12.8914 & 11.0631 \\
\hline 2 & Barbara & $\mathbf{1 0 . 6 7 8 5}$ & 13.142 & 11.2947 \\
\hline 3 & Boat & 8.3446 & 13.2356 & $\mathbf{7 . 3 5 5 2}$ \\
\hline 4 & Bridge & $\mathbf{1 1 . 3 0 4 8}$ & 14.0448 & 12.5354 \\
\hline 5 & Cameraman & 8.6786 & 9.9101 & $\mathbf{8 . 5 9 0 0}$ \\
\hline 6 & House & $\mathbf{1 1 . 2 7 4 5}$ & 12.5152 & 12.3645 \\
\hline 7 & Lena & $\mathbf{9 . 9 0 7 1}$ & 13.7681 & 10.3126 \\
\hline 8 & Mountain & $\mathbf{1 0 . 4 9 3 5}$ & 11.9843 & 10.5766 \\
\hline 9 & Peppers & $\mathbf{1 0 . 5 4 9 8}$ & 11.7984 & 11.0332 \\
\hline 10 & Rice & $\mathbf{1 2 . 7 1 5 7}$ & 13.6784 & 13.4477 \\
\hline 11 & Sailboat & $\mathbf{1 0 . 1 0 6 3}$ & 12.5904 & 10.6945 \\
\hline 12 & Zelda & $\mathbf{1 1 . 2 7 4 5}$ & 12.5152 & 12.3645 \\
\hline
\end{tabular}

\section{CONCLUSION}

Measures of uncertainty are proved to be effective tools for image segmentation. In this paper, new fuzzy rough uncertainty measures have been defined on fuzzy approximation spaces. A histogram based image thresholding algorithm using the fuzzy roughness measure is developed and experimented with different images. The segmented images of twelve test images using the proposed algorithm have been compared with those of OTSU method and FCM method. It is observed that the overlapping of the foreground background pixels in the images segmented using the proposed method is lesser than those of the other two methods. Further, the proposed algorithm produces images having the least root mean square error values in ten out of twelve cases. Thus, the proposed image segmentation algorithm can be used as an alternative for OTSU and FCM methods which are two popular methods used for binary segmentation.

\section{REFERENCES}

1. J. C. Bezdek, Pattern recognition with fuzzy objective function algorithms Plenum Press, New York, 2013.

2. L. D'eer, N. Verbiest, C. Cornelis, and L. Godo, "A comprehensive study of implicator conjunctor -based and noise-tolerant fuzzy rough sets: defnitions, properties and robustness analysis", Fuzzy Sets and Systems, Vol.275, 2015, pp 1-38

3. N. Dhanachandra and Y. J. Chanu, "A survey on image segmentation methods using clustering techniques", European Journal of Engineering Research and Science, Vol.2(1), 2017, pp 15-20.

4. D. Dubois and H. Prade, "Rough fuzzy sets and fuzzy rough sets", International Journal of General Systems, Vol.17(2-3), 1990, pp 191-209

5. C. A. Glasbey, An analysis of histogram-based thresholding algorithms", CVGIP: Graphical models and image processing, Vol.55(6), 1993, pp532-537.

6. A. E. Hassanien, A. Abraham, J. F. Peters, G. Schaefer, and C. Henry, "Rough sets and near sets in medical imaging: a review", IEEE Transactions on Information Technology in Biomedicine, Vol.13(6), 209, pp955-968.

7. R. Jacob and A. S. Kuriakose, A Study on Fuzzy Image Processing and Related Areas, PhD Dissertation, Mahatma Gandhi University, India (2015).

8. E. E. Kerre and M. Nachtegael(Eds), Fuzzy Techniques in Image Processing, Sringer-Verlag, Heidelberg (2000).

9. J. Klir and B. Yuan, Fuzzy Sets and Fuzzy Logic (Prentice Hall, New Jersey,1995)

10. J. Y. Liang, K. S. Chin, C. Y. Dang and R. C. M. Yam, "A New Method for Measuring Uncertainty and Fuzziness in Rough Set Theory", International Journal of General Systems 31 (4) (2002) 331- 342.

11. J.S. Mi, X. M. Li, H. Y. Zhao and T. Feng, "Information Theoretic Measures of Uncertainty in Generalized Fuzzy Rough Sets", in A. An et al. (eds) RSFDGrC 2007, LNAI 4482, (Springer 2007) 63 - 70.

12. J.S. Mi, Y. Leung and T. Feng, "Generalized Fuzzy Rough Sets Determined by a Triangular Norm", Information Sciences 178 (2008) 3203-3213.

13. S. Montes, I. Couso, P. Gil and C. Bertoluzza, " Divergence Measure Between Fuzzy Sets" International Journal of Approximate Reasoning 30 (2002) 91-105

14. N. Otsu, "A Threshold Selection Algorithm from Gray Level Histograms", IEEE Transactions on Systems, Man and Cybernetics 9 (1979) 62 - 66

15. Z. Pawlak, "Rough Sets", International Journal of Computer and Information Sciences 11(5), (1982) 341 - 356.

16. Z. Pawlak, Rough Sets - Theoretical Aspect of Reasoning About Data (Kluwer Academic Publishers,The Netherlands, 1991).

17. A. Radzikowska and E. E. Kerre, "A comparative Study of Fuzzy Rough Sets", Fuzzy Sets and Systems 126 (2002) 137-155.

18. N. Senthilkumaran and S. Vaithegi, "Image Segmentation by Using Thresholding Techniques for Medical Images", Computer Science and Engineering: An International Journal, 6(1) (2016)

19. C. E. Shannon, 'A Mathematical Theory of Communication", The Bell System Technical Journal 27 (1948) 379 - 423.

20. M. J. Wierman, Measuring Uncertainty in Rough Set Theory, International Journal of General System 28 (1999) 283 - 297.

21. W. Z. Wu, J. S. Mi, and W. X. Zhang, "Generalized fuzzy rough sets", Information sciences, 151:263\{282, 2003.

22. L. A. Zadeh,"Fuzzy Sets", Information and Control 8(3)(1965) 338-353.

23. J. Zhao, Z. Zhang, C. Han, and Z. Zhou, "Complement information entropy for uncertainty measure in fuzzy rough set and its applications", Soft Computing, 19 (7) (2015) 1997 - 2010.

\section{AUTHORS PROFILE}

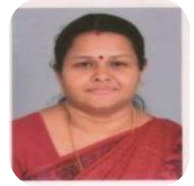

Mrs. Sheeja T. K. was awarded $\mathrm{PhD}$ degree by Mahatma Gandhi University, Kottayam in 2019. Her research interests include fuzzy set theory and rough set theory. She has published 5 research papers in international journals. She has fifteen years of teaching experience. She is presently working as an Assistant professor under the Collegiate Education Department of Govt. of Kerala.

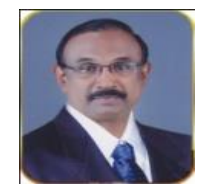

Dr. Sunny Kuriakose A. was awrded PhD degree by Cochin University of Science and Technology, Kerala, India in 1995. He has more than three decades of teaching experience. He is currently serving as the Dean and a Professor at Federal by Institute of Science and technology, Angamaly, Kerala, India. Sixteen scholars have been awarded $\mathrm{Ph} . \mathrm{D}$ degree under his supervision. He has published more than sixty research papers in various national and international journals. He authored two books and edited a number of volumes. His research interest includes Fuzzy Logic, Graph Theory, Decision Theory etc. He served the Kerala Mathematical Association as its General Secretary for eight years. Presently, he is the Academic Secretary of the Association.

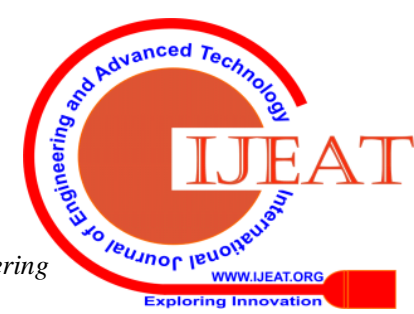

